\title{
Erratum for Glynn and Quinn (2010)
}

\author{
Adam N. Glynn \\ Department of Political Science, Emory University, 303 Tarbutton Hall, \\ 1555 Dickey Drive, Atlanta, GA 30322 \\ e-mail: adam.glynn@emory.edu (corresponding author) \\ Kevin M. Quinn \\ UC Berkeley School of Law, 490 Simon No 7200, Berkeley, CA 94720-7200 \\ e-mail:kquinn@law.berkeley.edu
}

In Glynn and Quinn (2010), equation (A3) of Appendix A read as

$$
\frac{(1-X) Y}{1-\pi\left(\mathbf{Z}, \psi^{*}\right)}=\frac{(1-X) Y(0)}{1-\pi\left(\mathbf{Z}, \psi^{*}\right)}=Y(0)+\frac{\left[X-\pi\left(\mathbf{Z}, \psi^{*}\right)\right] Y(0)}{1-\pi\left(\mathbf{Z}, \psi^{*}\right)} .
$$

It should have read as

$$
\frac{(1-X) Y}{1-\pi\left(\mathbf{Z}, \psi^{*}\right)}=\frac{(1-X) Y(0)}{1-\pi\left(\mathbf{Z}, \psi^{*}\right)}=Y(0)-\frac{\left[X-\pi\left(\mathbf{Z}, \psi^{*}\right)\right] Y(0)}{1-\pi\left(\mathbf{Z}, \psi^{*}\right)} .
$$

We thank Grace Fan and Erica Moodie for pointing us to this error.

\section{Reference}

Glynn, Adam N., and Kevin M. Quinn. 2010. An introduction to the augmented inverse propensity weighted estimator. Political Analysis 18(1):36-56. 